\title{
INCAPACIDADES FÍSICAS EM HANSENÍASE: CARACTERIZAÇÃO, FATORES RELACIONADOS E EVOLUÇÃO*
}

\author{
Gabriela de Cássia Ribeiro ${ }^{1}$, Francisco Carlos Félix Lana²
}

${ }^{1}$ Enfermeira. Doutoranda em Enfermagem. Docente de Enfermagem da Universidade Federal dos Vales do Jequitinhonha e Mucuri. Diamantina, MG, Brasil

${ }^{2}$ Enfermeiro. Doutor em Enfermagem. Docente da Escola de Enfermagem da Universidade Federal de Minas Gerais. Belo Horizonte, MG, Brasil.

RESUMO: Este estudo objetivou caracterizar os casos novos de hanseníase; analisar os aspectos socioeconômicos, demográficos, clínico-epidemiológicos e de acesso ao diagnóstico e tratamento relacionados à ocorrência de hanseníase com incapacidades físicas; e a evolução das incapacidades durante o tratamento. Foi realizada uma pesquisa de coorte retrospectiva na região de saúde de Diamantina-MG. Foram analisadas 107 notificações de hanseníase entre 2005 a 2010 e 71 entrevistas. A maioria dos diagnósticos foram multibacilares (73,2\%) e com incapacidade física $(79,1 \%)$. Houve relação estatística entre o desenvolvimento de deformidades físicas e os acometidos pela hanseníase com menor nível de escolaridade, com maior comprometimento neural e com maior dificuldade de deslocamento até a unidade de saúde, além de manutenção do grau de incapacidade física. Existe uma reduzida capacidade operacional da Estratégia de Saúde da Família quanto às Ações de Controle da Hanseníase e necessidade de implementação da política de acordo com o Sistema Único de Saúde.

DESCRITORES: Hanseníase; Epidemiologia; Pessoas com incapacidade física; Acesso aos serviços de saúde.

\section{PHYSICAL DISABILITIES IN LEPROSY: CHARACTERIZATION, FACTORS RELATED AND EVOLUTION}

ABSTRACT: The aim of this study was tocharacterize the new cases of leprosy; analyze socioeconomic, demographic, clinical-epidemiological, and access to diagnosis and treatment aspects related to the occurrence of leprosy with physical disabilities; and analyze the development of disabilities during treatment.A retrospective cohort survey was conducted in the health region of Diamantina, Minas Gerais, Brazil. One hundred seven reports of leprosy and 71 interviews were analyzed between 2005 and 2010. The most frequent diagnoses were multibacillary (73.2\%) and physical disability $(79.1 \%)$. There was a statistical relationship between the development of physical deformities and those affected by leprosy with lower levels of schooling, higher neural involvement, and greater difficulties in accessing the health unit, in addition to the maintenance of the degree of disability. There is a reduced operational capacity of the Family Health Strategy (FHS) regarding leprosy control actions and the need to implement the policy according to the Unified Health System (SUS, from its acronym in Portuguese).

DESCRIPTORS: Leprosy; Epidemiology; Disabled persons; Health services accessibility.

\section{INCAPACIDADES FÍSICAS EN ENFERMEDAD DE HANSEN: CARACTERIZACIÓN, FACTORES ASOCIADOS Y EVOLUCIÓN}

RESUMEN: Este estudio tuvo el objetivo de caracterizar los casos nuevos de enfermedad de Hansen; analizar los aspectos socioeconómicos, demográficos, clínicoepidemiológicos y de acceso al diagnóstico y tratamiento relacionados a la ocurrencia de enfermedad de Hansen con incapacidades físicas; y la evolución de las incapacidades durante el tratamiento. Fue realizada una investigación retrospectiva en la región de salud de Diamantina-MG. Fueron analizadas 107 notificaciones de enfermedad de Hansen entre 2005 y 2010, así como 71 entrevistas. La mayoría de los diagnósticos fue de multibacilares $(73,2 \%)$ y con incapacidad física $(79,1 \%)$. Hubo relación estadística entre el desarrollo de deformidades físicas y los acometidos por la enfermedad de Hansen con menor nivel de escolaridad, con mayor comprometimiento neural y con mayor dificultad de desplazamiento hasta la unidad de salud, además de manutención del grado de incapacidad física. Hay una reducida capacidad operacional de la Estrategia de Salud de la Familia cuanto a las Acciones de Control de Enfermedad de Hansen y necesidad de implementación de la política de acuerdo con el Sistema Único de Salud.

DESCRIPTORES: Enfermedad de Hansen; Epidemiología; Personas con incapacidad física; Acceso a los servicios de salud.

\footnotetext{
*Artigo extraído da dissertação de mestrado intitulada: "Fatores relacionados à prevalência de incapacidades físicas em Hanseníase na microrregião de Diamantina, Minas Gerais". Escola de Enfermagem da Universidade Federal de Minas Gerais, 2012.
} 


\section{INTRODUÇÃO}

A hanseníase possui grande importância para a Saúde Pública por se tratar de uma doença infectocontagiosa e crônica que atinge principalmente as pessoas em condições socioeconômicas precárias. Apresenta longo período de incubação, e há predileção do bacilo Mycobacterium leprae pelas células epiteliais e neurais, o que confere a doença um alto poder incapacitante ${ }^{(1)}$.

Entretanto, a hanseníase é uma doença curável com tratamento supervisionado por meio da Poliquimioterapia (PQT), podendo ser de 06 ou 12 meses dependendo da classificação operacional ${ }^{(2)}$.

Os pacientes de hanseníase são classificados quanto ao número de lesões cutâneas, carga bacilar e nível de acometimento dos nervos periféricos. Quando não há comprometimento neural são classificados como grau 0 de incapacidade física, o grau 1 de incapacidade ocorre quando há diminuição ou perda de sensibilidade nos olhos, mãos e pés e grau 2 de incapacidade quando há lesões mais graves nos olhos, mãos e pés ${ }^{(2)}$.

Estas complicações podem ser responsáveis por sequelas permanentes ao indivíduo, pois podem atingir os receptores nervosos responsáveis pela dor, visão e sensibilidade tátil, tornando-os mais susceptíveis a acidentes, queimaduras, feridas e, até mesmo, amputações, resultando em danos sociais e psíquicos que interferem na qualidade de vida ${ }^{(3)}$.

Neste sentido, a Organização Mundial de Saúde publicou em 2010, a Estratégia Global Aprimorada para Redução Adicional da Carga da Hanseníase: 2010-2015 e traçou como meta global para o ano de 2015, a redução em 35\% dos diagnósticos de hanseníase com grau 2 de incapacidade física por 100.000 habitantes, quando comparados com os dados apresentados em $2010^{(1,4)}$.

Para o sucesso e alcance da meta proposta, é necessário o diagnóstico precoce da hanseníase; o tratamento adequado, a vigilância de contatos e o acesso aos serviços públicos de saúde ${ }^{(5)}$.

Em 2013, o Brasil contabilizou 31.044 casos novos, apresentou coeficiente de detecção de $15,44 / 100.000$ habitantes, $64,4 \%$ dos casos novos foram diagnosticados como multibacilares e 7,3\% apresentavam grau 2 de incapacidade física ${ }^{(6)}$.

A região de saúde de Diamantina, estado de Minas Gerais (MG) apresentou no ano de 2012, 16 casos novos de hanseníase, taxa de detecção de 9,6/100.000 habitantes, no entanto $56,3 \%$ das notificações corresponderam aos diagnósticos com grau 2 de incapacidade física ${ }^{(7)}$.

Diante do grande número de diagnósticos tardios e com grau 2 de incapacidade física na região de saúde de Diamantina/MG, os objetivos dessa pesquisa foram caracterizar os casos novos de hanseníase na região no período de 2005 a 2010; analisar os aspectos socioeconômicos, demográficos, clínico-epidemiológicos e de acesso ao diagnóstico e tratamento da doença relacionados à ocorrência de hanseníase com incapacidades físicas, além de verificar a evolução das incapacidades nos momentos do diagnóstico e da alta medicamentosa.

\section{MÉTODO}

Foi realizado um estudo epidemiológico, tipo coorte retrospectiva, tendo como cenário a Região de Saúde de Diamantina, localizada no Vale do Jequitinhonha, Minas Gerais. A região possui 15 municípios e aproximadamente 167.000 habitantes.

O período delimitado para o estudo compreendeu os anos de 2005 a 2010. A escolha desta série histórica ocorreu em decorrência de uma característica específica das incapacidades físicas relacionadasàhanseníase, quesãoaltamente sensíveis à oscilação da capacidade operacional dos serviços de saúde, principalmente, no que tange à rotatividade profissional.

Em cinco dos municípios não houve diagnóstico de hanseníase neste período. A investigação foi realizada nas 10 cidades que fizeram notificações, que, a saber, são: Alvorada de Minas, Carbonita, Coluna, Couto de Magalhães de Minas, Datas, Diamantina, Gouveia, Itamarandiba, Presidente Kubitschek e Serro.

O banco de dados do Sistema de Informação de Agravos de Notificação (SINAN) - Hanseníase para análise das notificações foi disponibilizado pela Coordenadoria do Programa de Dermatologia Sanitária da Secretaria Estadual de Saúde e Superintendência Regional de Saúde de Diamantina.

Para a coleta de dados, utilizou-se um roteiro estruturado dividido em blocos. O primeiro bloco contemplou a análise das notificações do SINAN de casos novos de hanseníase, e os demais blocos estavam relacionados aos fatores socioeconômicos, demográficos, clínicoepidemiológicos e de acesso ao diagnóstico e 
tratamento da hanseníase.

Foram avaliadas 107 fichas de notificação do SINAN, sendo a amostra constituída por 71 entrevistados, 36 não participaram do estudo devido a mudança para cidades fora da região estudada (10), não localização do indivíduo (15) e óbito (11).

A coleta de dados ocorreu entre os meses de fevereiro e março de 2011. Os indivíduos foram contatados pelos profissionais da Estratégia de Saúde da Família (ESF) a que pertenciam, enquanto as entrevistas foram realizadas em suas residências ou nas respectivas unidades de saúde. Para as entrevistas, estima-se a duração de aproximadamente 25 minutos, e as mesmas não apresentaram recusa.

Como variável dependente, foi escolhido o grau de incapacidade física no diagnóstico. As variáveis independentes foram: as características socioeconômicas e demográficas - sexo, faixa etária, escolaridade, renda familiar e inserção no mercado de trabalho; os aspectos clínicoepidemiológicos - classificação operacional, forma clínica, modo de detecção, número de nervos acometidos e número de lesões cutâneas; o acesso ao diagnóstico e tratamento - procura pelos serviços de saúde após os primeiros sintomas, serviços que realizaram o diagnóstico e tratamento, tempo para realização do diagnóstico e início do tratamento, número de profissionais de saúde necessários para realizar o diagnóstico e dificuldade de deslocamento até a unidade de saúde; e a evolução da incapacidade - relação do grau de incapacidade no diagnóstico e na alta.

Foi construído um banco de dados no programa estatístico Epi Info, versão 6.0. Logo após a verificação de inconsistências, os dados foram exportados para o programa Microsoft Excel 2010 e, em seguida, para o programa estatístico SPSS versão 18.0 .

Realizou-se análise descritiva e bivariada. Os testes utilizados foram Qui-quadrado e Exato de Fisher para as variáveis categóricas e de KruskallWallis para as variáveis numéricas. Para avaliar a correlação entre o grau de incapacidade física nos momentos do diagnóstico e da alta, foi utilizado o de Teste de Homogeneidade Marginal. Foi verificado que todos eles tiveram como referência estatística o valor de $\mathrm{p}<0,05$.

Esta pesquisa foi submetida ao Comitê de Ética da Universidade Federal de Minas Gerais e aprovada com o parecer número ETIC 0512.0.203.000-10, em novembro de 2010. Todos os participantes foram orientados sobre os objetivos da pesquisa e, após autorização, assinaram um Termo de Compromisso Livre e Esclarecido. Por conseguinte, ficou assegurada aos participantes a garantia de anonimato e sigilo das informações.

\section{RESULTADOS}

Foram analisadas 107 notificações de casos novos de hanseníase e o município de Diamantina apresentou a maior proporção dos casos, sendo $47,7 \% \quad(n=51)$, seguido pelos municípios de Presidente Kubitschek, Serro e Coluna com $11,2 \% \quad(n=12), 10,3 \% \quad(n=11)$ e $9,3 \%$ $(n=10)$, respectivamente. A taxa de detecção de casos novos encontrada no período de estudo foi de 12,3/100.000 habitantes.

Para análise dos fatores que se relacionam com a presença de diagnósticos com incapacidades físicas, foram utilizadas as informações das 71 entrevistas realizadas junto aos indivíduos da Região de Saúde de Diamantina.

A Tabela 1 demonstra a relação entre os aspectos clínico-epidemiológicos no momento do diagnóstico de hanseníase e presença de incapacidades físicas.

Tabela 1 - Relação entre os aspectos clínicoepidemiológicos e incapacidades físicas. Região de Saúde de Diamantina, MG, Brasil, 2005-2010

\begin{tabular}{|c|c|c|c|c|}
\hline & \multicolumn{3}{|c|}{$\begin{array}{l}\text { Grau de incapacidade } \\
\text { física no diagnóstico }\end{array}$} & \multirow{3}{*}{$p$} \\
\hline & Grau 0 & Grau 1 & Grau 2 & \\
\hline & $\mathrm{N}(\%)$ & $\mathrm{N}(\%)$ & N (\%) & \\
\hline \multicolumn{5}{|l|}{ Forma clínica } \\
\hline Indeterminada & $4(26,7)$ & $9(60)$ & $2(13,3)$ & \\
\hline Tuberculoide & $2(66,7)$ & - & $1(33,3)$ & $0,050^{* *}$ \\
\hline Dimorfa & $4(10,5)$ & $19(50)$ & $\begin{array}{c}15 \\
(39,5) \\
\end{array}$ & \\
\hline Virchowiana & $4(36,4)$ & $5(45,4)$ & $2(18,2)$ & \\
\hline \multicolumn{5}{|c|}{ Classificação operacional } \\
\hline PB & $6(31,6)$ & $11(57,9)$ & $2(10,5)$ & $0,107^{*}$ \\
\hline $\mathrm{MB}$ & $9(17,3)$ & $25(48,1)$ & $\begin{array}{c}18 \\
(34,6) \\
\end{array}$ & \\
\hline \multicolumn{5}{|c|}{ Modo de detecção } \\
\hline Passiva & $\begin{array}{c}12 \\
(21,8) \\
\end{array}$ & $\begin{array}{c}30 \\
(54,5) \\
\end{array}$ & $\begin{array}{c}13 \\
(23,6) \\
\end{array}$ & $0,498 * *$ \\
\hline Ativa & $1(11,2)$ & $4(44,4)$ & $4(44,4)$ & \\
\hline
\end{tabular}

Foi possível identificar, um grande número de diagnósticos realizados nas formas multibacilares 
da hanseníase, 73,2\% e, destes, a forma clínica dimorfa foi a mais recorrente, seguida pelos virchowianos, correspondendo respectivamente a $73,1 \%$ e $21,2 \%$. Seguindo esta tendência, $79,1 \%$ dos casos apresentaram algum tipo de incapacidade física ao diagnóstico, chamando atenção para os que tiveram o grau 2 de incapacidade, $29,9 \%$.

A forma de detecção passiva foi a mais frequente, em que $77,5 \%$ dos casos foram identificados pelos encaminhamentos e demanda espontânea.

Houve uma relação estatística significativa entre a mediana de nervos acometidos no diagnóstico e o grau 2 de incapacidade $(p=0,006)$, conforme apresentado na Tabela 2.

Tabela 2 - Relação entre mediana de nervos acometidos, lesões cutâneas, contatos examinados e incapacidades físicas. Região de Saúde de Diamantina, MG, Brasil, 2005-2010

\begin{tabular}{|c|c|c|c|c|}
\hline & \multicolumn{3}{|c|}{$\begin{array}{c}\text { Grau de incapacidade física no } \\
\text { diagnóstico }\end{array}$} & \multirow{3}{*}{$p^{*}$} \\
\hline & Grau 0 & Grau 1 & Grau 2 & \\
\hline & $\begin{array}{l}\text { Mediana } \\
\text { (Min- } \\
\text { Max) }\end{array}$ & $\begin{array}{c}\text { Mediana } \\
\text { (Min- } \\
\text { Max) }\end{array}$ & $\begin{array}{l}\text { Mediana } \\
\text { (Min- } \\
\text { Max) }\end{array}$ & \\
\hline $\begin{array}{l}\text { Número } \\
\text { de nervos } \\
\text { acometidos }\end{array}$ & $0(0-4,5)$ & $2(1-4)$ & $\begin{array}{c}4(2,5- \\
7,5)\end{array}$ & 0,006 \\
\hline $\begin{array}{l}\text { Número } \\
\text { de lesões } \\
\text { cutâneas }\end{array}$ & $4(1-7)$ & $5(2-6)$ & $6(4-6)$ & 0,552 \\
\hline $\begin{array}{l}\text { Número de } \\
\text { contatos } \\
\text { examinados }\end{array}$ & $\begin{array}{c}3(1,7- \\
6,5)\end{array}$ & $3(1-4,5)$ & $2(1-5)$ & 0,739 \\
\hline
\end{tabular}

Em complemento a esse achado, verifica-se uma mediana elevada de lesões cutâneas (6) e reduzida de contatos examinados (2) dentre os que apresentaram grau 2 de incapacidade física.

A Tabela 3 nos mostra a relação entre o nível de instrução dos acometidos pela hanseníase e o desenvolvimento de deformidades físicas $(p=$ 0,032 ), uma vez que todos que apresentaram grau 2 de incapacidade possuíam menor escolaridade.

Os jovens e adultos (57,5\%); aqueles inseridos no mercado de trabalho (56,3\%); e os com renda familiar menor que 01 salário mínimo (52,2\%) foram majoritariamente acometidos pelo grau 1 de incapacidade física.

A representatividade do sexo feminino
Tabela 3 - Relação entre as características socioeconômicas e demográficas e incapacidades físicas. Região de Saúde de Diamantina, MG, Brasil, 2005-2010

\begin{tabular}{|c|c|c|c|c|}
\hline & \multicolumn{3}{|c|}{$\begin{array}{c}\text { Grau de incapacidade física } \\
\text { no diagnóstico }\end{array}$} & \multirow{3}{*}{$p$} \\
\hline & Grau 0 & Grau 1 & Grau 2 & \\
\hline & N (\%) & $\mathrm{N}(\%)$ & $\mathrm{N}(\%)$ & \\
\hline \multicolumn{5}{|l|}{ Escolaridade } \\
\hline $\begin{array}{r}\text { Sem/ensino } \\
\text { fundamental } \\
\text { incompleto }\end{array}$ & $11(18,6)$ & $\begin{array}{c}28 \\
(47,5)\end{array}$ & $\begin{array}{c}20 \\
(33,9)\end{array}$ & $0,032^{* *}$ \\
\hline $\begin{array}{l}\text { Fundamental } \\
\text { completo ou } \\
\text { mais }\end{array}$ & $4(33,3)$ & $8(66,7)$ & - & \\
\hline \multicolumn{5}{|l|}{ Faixa etária } \\
\hline 18 a 59 anos & $8(20)$ & $\begin{array}{c}23 \\
(57,5) \\
\end{array}$ & $9(22,5)$ & $0,380^{*}$ \\
\hline $\begin{array}{l}60 \text { anos e } \\
\text { mais }\end{array}$ & $7(22,6)$ & $\begin{array}{c}13 \\
(41,9) \\
\end{array}$ & $\begin{array}{c}11 \\
(35,5)\end{array}$ & \\
\hline \multicolumn{5}{|l|}{ Sexo } \\
\hline Feminino & $7(18,9)$ & $\begin{array}{c}21 \\
(56,8) \\
\end{array}$ & $9(24,3)$ & $0,565^{*}$ \\
\hline Masculino & $8(23,5)$ & $\begin{array}{c}15 \\
(44,1) \\
\end{array}$ & $\begin{array}{c}11 \\
(32,4) \\
\end{array}$ & \\
\hline \multicolumn{5}{|c|}{ Inserção mercado de trabalho } \\
\hline Sim & $6(18,7)$ & $\begin{array}{c}18 \\
(56,3) \\
\end{array}$ & $8(25)$ & $0,699 *$ \\
\hline Não & $9(23,1)$ & $\begin{array}{c}18 \\
(46,2) \\
\end{array}$ & $\begin{array}{c}12 \\
(30,7) \\
\end{array}$ & \\
\hline \multicolumn{5}{|l|}{ Renda familiar } \\
\hline $\begin{array}{l}\leq 1 \text { salário } \\
\text { mínimo }\end{array}$ & $4(17,4)$ & $\begin{array}{c}12 \\
(52,2) \\
\end{array}$ & $7(30,4)$ & $0,860^{*}$ \\
\hline $\begin{array}{l}1 \text { a } 3 \text { salários } \\
\text { mínimos }\end{array}$ & $11(23)$ & $24(50)$ & $13(27)$ & \\
\hline
\end{tabular}

$(52,1 \%)$ se mostrou ligeiramente maior que a do sexo masculino que foi de $47,9 \%$. Entretanto, os homens obtiveram mais diagnósticos de grau 2 de incapacidade física $(32,4 \%)$.

Os entrevistados que relataram problemas de deslocamento até as unidades de saúde (Tabela 4), devido a condição física, foram diagnosticados em sua maioria com grau 2 de incapacidade física, demonstrando significância estatística $(p=0,031)$.

Apenas 56 notificações continham informações sobre a avaliação do grau de incapacidade no momento da alta (Tabela 5).

A análise da evolução das incapacidades demonstra um valor muito próximo a significância estatística $(p=0,050)$ que há uma tendência para a 
Tabela 4 - Relação entre acesso ao diagnóstico e tratamento e incapacidades físicas. Região de Saúde de Diamantina/MG, 2005-2010

$\begin{gathered}\text { Grau de incapacidade física } \\
\text { no diagnóstico }\end{gathered}$
\begin{tabular}{ccc}
\hline Grau 0 & Grau 1 & Grau 2 \\
\hline N (\%) & N (\%) & N (\%)
\end{tabular}

Dificuldade de deslocamento até unidade de saúde

\begin{tabular}{|c|c|c|c|c|}
\hline Sim & $7(21,2)$ & $12(36,4)$ & $14(42,4)$ & 0,031 \\
\hline Não & $8(21,1)$ & $24(63,2)$ & $6(15,7)$ & \\
\hline \multicolumn{5}{|c|}{ Local que realizou o diagnóstico } \\
\hline ESF/EACS & $7(15,6)$ & $25(55,6)$ & $13(28,9)$ & 0,301 \\
\hline Outros & $8(30,8)$ & $11(42,3)$ & $7(26,9)$ & \\
\hline \multicolumn{5}{|c|}{ Local que realizou o tratamento } \\
\hline ESF/EACS & $9(18,4)$ & $26(53,1)$ & $\begin{array}{c}14 \\
(28,5)\end{array}$ & 0,686 \\
\hline Outros & $6(27,3)$ & $10(45,4)$ & $6(27,3)$ & \\
\hline
\end{tabular}

Tempo entre procura e primeira consulta

\begin{tabular}{|c|c|c|c|c|}
\hline 1 a 4 dias & $9(20)$ & $25(55,6)$ & $11(24,4)$ & 0,535 \\
\hline 5 dias ou & $6(23,1)$ & $11(42,3)$ & $9(34,6)$ & \\
\hline
\end{tabular}

Consultas para realizar o diagnóstico

\begin{tabular}{|c|c|c|c|c|}
\hline 1 a 3 & $\begin{array}{c}11 \\
(20,4) \\
\end{array}$ & $28(51,9)$ & $15(27,8)$ & 0,936 \\
\hline 4 ou mais & $4(23,5)$ & $8(47,1)$ & $5(29,4)$ & \\
\hline \multicolumn{5}{|c|}{ Tempo para realizar o diagnóstico } \\
\hline $\begin{array}{l}\text { Imediato a } 6 \\
\text { meses }\end{array}$ & $13(21,1)$ & $31(50,1)$ & $17(27,8)$ & $1,000^{* *}$ \\
\hline \begin{tabular}{ll}
\multicolumn{2}{c}{$\begin{array}{l}\text { meses } \\
\text { mais de }\end{array}$} \\
ano
\end{tabular} & $2(20)$ & $5(50)$ & $3(30)$ & \\
\hline
\end{tabular}

Tempo entre diagnóstico e início do tratamento

\begin{tabular}{ccccc}
$\begin{array}{c}\text { Imediato a } \\
30 \text { dias }\end{array}$ & $\begin{array}{c}11 \\
(24,4)\end{array}$ & $21(46,7)$ & $13(27,9)$ & $0,375^{* *}$ \\
$\begin{array}{c}\text { Mais de } 30 \\
\text { dias }\end{array}$ & $1(7,1)$ & $9(64,3)$ & $4(28,6)$ & \\
\hline
\end{tabular}

Número de profissionais de saúde que atenderam até o diagnóstico

\begin{tabular}{lcccc}
\hline 1 & $2(11,8)$ & $10(58,8)$ & $5(29,4)$ & $0,623^{* *}$ \\
\hline 2 ou mais & 13 & $26(48,1)$ & $15(27,8)$ & \\
& $(24,1)$ & & &
\end{tabular}

ESF/EACS Estratégia de Saúde da Família e da Estratégia de Agentes Comunitários de Saúde

*Teste do Qui-quadrado; **Teste de Fisher.

manutenção do grau de incapacidade entre os momentos do diagnóstico e da alta.

\section{DISCUSSÃO}

A taxa de detecção de casos novos de hanseníase apresentada pelos municípios de estudo, é considerada alta segundo os parâmetros
Tabela 5 - Evolução dos graus de incapacidade física entre os momentos do diagnóstico e da alta. Região de Saúde de Diamantina, MG, Brasil, 2005-2010

\begin{tabular}{|c|c|c|c|c|}
\hline & \multicolumn{4}{|c|}{ Incapacidade física na alta } \\
\hline & $\begin{array}{c}\text { Grau } 0 \\
(\%)\end{array}$ & $\begin{array}{c}\text { Grau } 1 \\
(\%)\end{array}$ & $\begin{array}{l}\text { Grau } 2 \\
(\%)\end{array}$ & $p^{*}$ \\
\hline \multicolumn{5}{|c|}{ Incapacidade no diagnóstico } \\
\hline Grau 0 & $7(53,8)$ & $6(46,2)$ & - & \multirow{3}{*}{0,05} \\
\hline Grau 1 & $12(41,4)$ & $14(48,3)$ & $3(10,3)$ & \\
\hline Grau 2 & $2(14,3)$ & $4(28,6)$ & $8(57,1)$ & \\
\hline
\end{tabular}

*Teste de Homogeneidade Marginal.

do Ministério da Saúde ${ }^{(2)}$. Apesar desta região se configurar endêmica para hanseníase, existem municípios que por vários anos seguidos não realizam diagnósticos.

O conhecimento da distribuição espacial da hanseníase e seu comportamento epidemiológico são primordiais para que se possa intensificar as ações de vigilância em saúde e identificar prioridades no sentido de controlar a endemia ${ }^{(8)}$.

O alto percentual de casos notificados de hanseníase com grau 2 de incapacidade física e o predomínio das formas multibacilares, também foram encontrados em estudo realizado em outra região endêmica do Vale do Jequitinhonha, o que reforça a afirmativa da persistência do ciclo da doença na região e consequentemente, a prevalência oculta ${ }^{(9)}$.

Outro fator contribuinte para o diagnóstico não oportuno da hanseníase é a predominância das formas passivas de detecção. $\mathrm{O}$ modelo de atenção a saúde ainda vigente e praticado pelos serviços da Atenção Primária à Saúde (APS), prioriza o atendimento ao indivíduo, deixando em segundo plano as ações coletivas e em grupo(5), contrariando o que preconiza a Política Nacional de Atenção Básica (PNAB) ${ }^{(10)}$.

Ainda é necessário avançar, pois a busca ativa dos casos de hanseníase consiste em uma importante ferramenta para diagnóstico precoce e redução das possíveis complicações da doença ${ }^{(2,11)}$. Principalmente em áreas de maior densidade populacional, onde se verifica a necessidade de se estender a busca ativa para os contatos domiciliares e sociais do caso-índice da hanseníase ${ }^{(11)}$.

Os resultados relacionados à presença de incapacidade física dentre aqueles que possuem maior número de nervos acometidos vem ao encontro dos achados de um estudo realizado no estado de Bengala na Índia ${ }^{(12)}$. É premissa básica 
a avaliação dermatoneurológica do paciente no momento do diagnóstico e ao longo do tratamento, pois há maior preocupação por parte dos profissionais de saúde com as lesões de pele apresentadas pelo portador da hanseníase, esquecendo-se do componente neurológico da doença e principalmente daqueles classificados como neural puro ${ }^{(12)}$.

Neste estudo, os homens apresentaram maior número de diagnósticos de hanseníase com complicações do que as mulheres, configurando uma detecção mais tardia. Este fato pode ser explicado pela grande atenção dispensada pelas mulheres à estética corporal e pela predominância de programas voltados para a saúde da mulher ${ }^{(13)}$. Nesse sentido, o acesso dos homens aos serviços da APS limita-se ao tratamento de uma morbidade $^{(14)}$.

Houve uma relação significativa entre o menor nível de instrução e o acometimento pelo grau 2 de incapacidade física. A baixa escolaridade é um forte indicador de condições precárias de moradia, nutrição, higiene e acesso aos serviços de saúde ${ }^{(15)}$, fatores determinantes para a manutenção da cadeia de transmissão da hanseníase.

Independentemente do nível de escolaridade, é por meio da educação em saúde que as pessoas serão sensibilizadas quanto aos primeiros sinais da hanseníase, a necessidade de diagnóstico precoce e prevenção de incapacidades. Os diversos espaços comunitários podem ser utilizados para disseminar tal informação, como reuniões em grupo, sala de espera, propagandas televisivas e radiofônicas ${ }^{(16)}$.

A ampliação de espaços de escuta para portadores de hanseníase, principalmente na APS, constitui-se um desafio para o enfrentamento da doença, pois os profissionais de saúde tendem a desenvolver ações voltadas para o adoecimento e terapêutica em detrimento das preventivas. Estes locais necessitam ser verdadeiramente significativos para os portadores de hanseníase, suas famílias e comunidade para que possam sanar suas dúvidas e compartilhar suas experiências prévias $^{(17)}$.

As características socioeconômicas da população estudada compõem o quadro de vulnerabilidade social apresentado. Considerando que a maioria dos entrevistados eram jovens e adultos com renda familiar baixa, pode-se inferir que estavam inseridos no mercado de trabalho em ocupações manuais e pesadas, as quais quando congregadas ao poder incapacitante da hanseníase levam a uma futura população de inativos. Aqueles que conseguem permanecer no mercado de trabalho, precisam se adaptar a novas funções compatíveis com a sua condição de saúde, sujeitando-se muitas vezes, a redução da renda mensal| ${ }^{(18,3)}$.

Concomitante a isso, estas pessoas podem ficar mais vulneráveis ao aparecimento de lesões em áreas periféricas, especialmente nos pés ${ }^{(12)}$, comprometendo seu deslocamento conforme demonstrado neste estudo. Os pacientes com doenças crônicas desenvolvem problemas de locomoção e se tornam dependentes de um meio de transporte que os levem até a unidade de saúde e normalmente precisam pagar por ele ${ }^{(19)}$. Essa situação pode determinar o abandono do tratamento, permanência do estigma gerado pela doença e redução de qualidade de vida.

A ESF destacou-se como porta de entrada para a realização de tratamento, revelando um movimento ainda que rudimentar, no sentido da descentralização das Ações de Controle da Hanseníase $(\mathrm{ACH})$. Tais ações de prevenção e controle da doença estão inseridas nas atividades da APS e, recomenda-se, inclusive, que sejam incorporadas a outras políticas públicas de saúde com a finalidade de se reduzir os danos causados por suas complicações ${ }^{(2,20)}$.

A descentralização das $\mathrm{ACH}$, não exclui o atendimento ao portador de hanseníase nos níveis de maior complexidade, dependendo de sua condição clínica e em casos de recidiva ${ }^{(21)}$. Entretanto, devido à desorganização do processo de trabalho voltado para a assistência ao portador de hanseníase na APS, observa-se que muitas unidades realizam apenas o diagnóstico e encaminham o paciente para tratamento nas unidades de referência ${ }^{(22)}$. Tal fluxo que se observa também entre municípios ${ }^{(21)}$. Este fato é frequente na Policlínica Regional de Diamantina, que além de outras atribuições, funciona como Centro de Referência para hanseníase.

Outras evidências sugerem a detecção tardia da hanseníase, pois alguns pacientes relataram que foram diagnosticados após sete meses de consultas subsequentes e a maioria precisou ser atendida por dois ou mais profissionais de saúde para concluir o caso. Apesar desse estudo não demonstrar significância estatística para essas variáveis, sabe-se que estes eventos são suficientes para a evolução dos sintomas e aparecimento dos comprometimentos físicos. 
Evidenciou-se a negligência da informação sobre a avaliação do grau de incapacidade no momento da alta. Tal situação revela que os serviços priorizam a avaliação ao diagnóstico e pormenorizam o acompanhamento deste dado durante o tratamento, impedindo uma análise apurada da progressão do grau de incapacidade física ${ }^{(9)}$. A falta de informação na notificação de hanseníase compromete as ações de vigilância epidemiológica ${ }^{(21)}$, bem como coloca em discussão a qualidade da assistência prestada.

Assim, a análise da evolução das incapacidades revela uma tendência à manutenção do grau de incapacidade nos momentos do diagnóstico e da alta corroborando os achados em outros municípios do Vale do Jequitinhonha $(M G)^{(9)}$ e do Tocantins ${ }^{(22)}$.

Neste sentido, é mandatório que as ações de prevenção de incapacidades ocorram paralelamente às medidas de controle da doença como a realização frequente do exame dermatoneurológico ${ }^{(13)}$. Por conseguinte, os gestores e profissionais de saúde necessitam compreender a hanseníase como condição crônica de saúde(22), para que as $\mathrm{ACH}$ sejam efetivas e disponíveis desde o momento do diagnóstico, durante o tratamento e no pós-alta medicamentosa.

\section{CONCLUSÃO}

Este estudo apresentou como limitação o reduzido número amostral, bem como a impossibilidade de análise de algumas informações devido às falhas de preenchimento das fichas do SINAN.

Entretanto, atingiu seus objetivos, pois a análise dos resultados revela: o baixo nível de escolaridade dos entrevistados, a presença de nervos acometidos no momento do diagnóstico e a dificuldade de deslocamento até a unidade de saúde devido às limitações físicas, como fatores relacionados à ocorrência dos diagnósticos com grau 2 de incapacidade física na Região de Saúde de Diamantina. Da mesma forma, verificou-se que não existem ações para redução do grau de incapacidade entre os momentos do diagnóstico e da alta medicamentosa.

A ESF, porta de entrada para os pacientes de hanseníase, ainda apresenta uma capacidade operacional reduzida no que tange às Ações de Controle da Hanseníase. Há necessidade de os gestores planejarem ações de hanseníase nos municípios de acordo com a política pública vigente e implementarem os preceitos do Sistema Único de Saúde.

Ressalta-se também a necessidade de ampliação do campo de pesquisas em hanseníase nesta região, no sentido de se conhecer o comportamento epidemiológico da doença e reduzir os diagnósticos tardios com presença de incapacidades físicas.

\section{REFERÊNCIAS}

1. Organização Mundial da Saúde. Estratégia global aprimorada para redução adicional da carga da hanseníase (Período do plano: 2011-2015). Organização PanAmericana de Saúde. 2010. [acesso em 20 jun 2015]. Disponível: http://www.paho.org/ $\mathrm{bra} /$ index.php?option $=\mathrm{com}_{-}$docman\&task $=\mathrm{doc}_{-}$ view\&gid $=1044 \&$ Itemid $=423$

2. Ministério da Saúde (BR). Portaria $\mathrm{n}^{\circ} 3.125$, de 07 de outubro de 2010. Aprova as Diretrizes para Vigilância, Atenção e Controle da Hanseníase. Diário Oficial da União, [Internet] 15 out 2010 [acesso em 20 jun 2015]. Disponível: http://bvsms.saude.gov.br/bvs/saudelegis/ gm/2010/prt3125_07_10_2010.html

3. Seshadri D, Khaitan BK, Khanna N, Sagar R. Dehabilitation in the era of elimination and rehabilitation: a study of 100 leprosy patients from a tertiary care hospital in India. Lepr Rev. [Internet] 2015;86(1) [acesso em 20 jun 2015]. Disponível: http:// www.lepra.org.uk/platforms/lepra/files/lr/Mar15/1850. pdf

4. Chhabra N, Grover C, Singal A, Bhattacharya SN, Kaur R. Leprosy scenario at a tertiary level hospital in Delhi: A 5-year retrospective study Year. Indian J Dermatol [Internet] 2015;60(1) [acesso em 20 jun 2015]. Disponível: http://www.e-ijd.org/text. asp?2015/60/1/55/147793

5. Lanza FM, Lana FCF, Carvalho APM, Davi RFL. Ações de controle da hanseníase: tecnologias desenvolvidas nos municípios do Vale do Jequitinhonha, Minas Gerais. R. Enferm. Cent. O. Min. [Internet] 2011;1(2) [acesso em 20 jun 2015] Disponível: http://www.seer. ufsj.edu.br/index.php/recom/article/view/82/136

6. Ministério da Saúde (BR). Secretaria de Vigilância em Saúde. Departamento de Vigilância Epidemiológica. Coordenação Geral de Hanseníase e Doenças em Eliminação. Registro ativo: número e percentual, Casos novos de hanseníase: número, coeficiente e percentual, faixa etária, classificação operacional, sexo, grau de incapacidade, contatos examinados, por estados e regiões. 2013. [acesso em 20 jun 2015]. Disponível: http://portalsaude.saude.gov.br/images/ pdf/2014/dezembro/01/Dados-2013.pdf

7. Secretaria de Estado da Saúde de Minas Gerais. 
Seminário Estadual de Monitoramento e Avaliação em Hanseníase Minas Gerais 2012. Belo Horizonte: Secretaria de Estado da Saúde; 2013.

8. Garcia DR, Ignotti E, Cortela DCB, Xavier DR, Barelli Carla SGAP. Análise espacial dos casos de hanseníase, com enfoque à área de risco, em uma unidade básica de saúde no município de Cáceres (MT) Cad. Saúde Coletiva. [Internet] 2013;21(2) [acesso em 20 jun 2015]. Disponível: http://dx.doi.org/10.1590/S1414462X2013000200011

9. Lana FCF, Carvalho APM, Davi RFL. Perfil epidemiológico da hanseníase na microrregião Araçuaí. Esc. Anna Nery. [Internet] 2011;15(1) [acesso em 20 jun 2015]. Disponível: http://dx.doi.org/10.1590/ S1414-81452011000100009

10. Ministério da Saúde (BR). Secretaria de Atenção à Saúde. Departamento de Atenção Básica. Política Nacional de Atenção Básica. Brasília: Ministério da Saúde, 2012. [acesso em 20 jun 2015]. Disponível: http://189.28.128.100/dab/docs/publicacoes/geral/ pnab.pdf.

11. Moura MLN, Dupnik KM, Sampaio GAA, Nóbrega PFC, Jeronimo AK, Nascimento-Filho JM et al. Active Surveillance of Hansen's Disease (Leprosy): Importance for Case Finding among Extra-domiciliary Contacts. PLoS Negl Trop Dis. [Internet] 2013;7(3) [acesso em 20 jun 2015]. Disponível: http://dx.doi. org/10.1371/journal.pntd.0002093

12. Sarkar J, Dasgupta A, Dutt D. Disability among new leprosy patients, an issue of concern: An institution based study in an endemic district for leprosy in the state of West Bengal, India. Indian J Dermatol Venereol Leprol. [Internet] 2012;78(3) [acesso em 20 jun 2015]. Disponível: http://dx.doi.org/10.4103/0378-6323.95449

13. Araújo e Araújo AER, Aquino DMC, Goulart IMB, Pereira SRF, Figueiredo IA, Serra $\mathrm{HO}$ et al. Factors associated with neural alterations and physical disabilities in patients with leprosy in São Luis, State of Maranhão, Brazil. Rev. Soc. Bras. Med. Trop. [Internet] 2014;47(4) [acesso em 20 jun 2015]. Disponível: http:// dx.doi.org/10.1590/0037-8682-0119-2014

14. Silva DM, Souza TO, Lima MV, Yarid SD, Sena ELS. Acessibilidade do homem aos serviços da atenção básica: uma aproximação com a bioética da proteção. Cogitare enferm. 2013;18(3):573-8.

15. Lustosa AA, Nogueira LT, Pedrosa JIS, Teles JBM, Campelo V. The impact of leprosy on health-related quality of life. Rev. Soc. Bras. Med. Trop. [Internet] 2011;44(5) [acesso em 20 jun 2015]. Disponível: http:// dx.doi.org/10.1590/S0037-86822011000500019.

16. Moreira AJ, Naves JM, Fernandes LFRM, Castro SS, Walsh IP. Ação educativa sobre hanseníase na população usuária das unidades básicas de saúde de
Uberaba-MG. Saúde debate [Internet]. 2014;38(101) [acesso em 20 jun 2015]. Disponível: http://dx.doi. org/10.5935/0103-1104.20140021.

17. Silva MCD, Paz EPA. Educação em saúde no programa de controle da hanseníase: a vivência da equipe multiprofissional. Esc. Anna Nery. [Internet] 2010;14(2) [acesso em 20 jun 2015]. Disponível: http:// dx.doi.org/10.1590/S1414-81452010000200003

18. Smith N. The face of disability in Nigeria: a disability Survey in Kogi and Niger states. Disability, CBR and Inclusive Development. [Internet] 2011;22(1) [acesso em 20 jun 2015]. Disponível: http://dx.doi.org/10.5463/ dcid.v22i1.11

19. Arantes CK, Garcia MLR, Filipe MS, Nardi SMT, Paschoal VD. Avaliação dos serviços de saúde em relação ao diagnóstico precoce da hanseníase. Epidemiol. Serv. Saúde [Internet] 2010;19(2) [acesso em 20 jun 2015]. Disponível: http://scielo.iec.pa.gov.br/ pdf/ess/v19n2/v19n2a08.pdf

20. Lanza, FM, Lana, FCF. Descentralização das ações de controle da hanseníase na microrregião de Almenara, Minas Gerais. Rev. Latino-Am. Enfermagem [Internet] 2011;19(1) [acesso em 20 jun 2015]. Disponível: http:// dx.doi.org/10.1590/S0104-11692011000100025

21. Alencar CHM, Ramos Jr. AN, Sena NSA, Murto C, AlencarMJF, Barbosa JCetal. Diagnóstico da hanseníase fora do município de residência: uma abordagem espacial, 2001 a 2009. Cad. Saúde Pública [Internet] 2012;28(9) [acesso em 20 jun 2015]. Disponível: http:// dx.doi.org/10.1590/S0102-311X2012000900008.

22. Monteiro LD, Alencar CHM, Barbosa JC, Braga KP, Castro MD, Heukelbach J. Incapacidades físicas em pessoas acometidas pela hanseníase no período pósalta da poliquimioterapia em um município no Norte do Brasil. Cad. Saúde Pública [Internet] 2013;29(5) [acesso em 20 jun 2015]. Disponível: http://dx.doi. org/10.1590/S0102-311X2013000500009. 